\begin{tabular}{|c|c|c|}
\hline 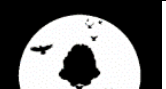 & & $\begin{array}{l}\text { ISSN: 1991-2951 (Print) } \\
\text { ISSN: 2091-2781 (Online) }\end{array}$ \\
\hline $\begin{array}{l}\text { Nature Conservation \& } \\
\text { Heallh Care Council } \\
\text { Ellatinogat, Nepall }\end{array}$ & Journal homepage: http://nepjol.info/index.php/ON & (c) ${ }_{\mathrm{BY}}$ (i) \\
\hline
\end{tabular}

\title{
Application of Water Quality Index (WQI) for groundwater quality assessment of Biratnagar, Nepal
}

\author{
Bishnu Dev Das $^{1 *}$ and Sunil Kumar Choudhary ${ }^{2}$ \\ ${ }^{1}$ Department of Botany, Mahendra Morang Aadarsh Multiple Campus, Tribhuvan University, Biratnagar, Nepal \\ ${ }^{2}$ University Department of Botany, Tilka Manjhi Bhagalpur University, Bhagalpur - 812 007, India \\ *E-mail: bishnudevnp@gmail.com
}

\begin{abstract}
This present hydrological study assessed the groundwater quality of Biratnagar Metropolitan by the application of Water Quality Index (WQI). It has been determined on the basis of analyzed groundwater samples for some important physicochemical parameters such as $\mathrm{pH}$, turbidity, electrical conductivity (EC), dissolved oxygen (DO), free carbon-dioxide $\left(\mathrm{FCO}_{2}\right)$, bicarbonate $\left(\mathrm{HCO}_{3}\right)$, total hardness $(\mathrm{TH})$, phosphate $\left(\mathrm{PO}_{4}-\mathrm{P}\right)$, nitrate- $\mathrm{N}\left(\mathrm{NO}_{3}-\mathrm{N}\right)$, arsenic $(\mathrm{As})$, and fluoride $(\mathrm{Fl})$ collected from 110 representative tube wells during post-monsoon period of 2015. The status of fluoride was below detectable level (BDL) in all the analyzed groundwater samples. The WQI for these samples ranged from 84.54 to 403.14. The high value of WQI has been found mainly due to the higher values of turbidity, $\mathrm{FCO}_{2}$ and arsenic in the groundwater. The results of study have been used to recommend models for predicting water quality. The classification of water quality on the basis of WQI value have been found to be good water $(18.18 \%)$, poor water $(59.09 \%)$, very poor water $(13.64 \%)$ and unsuitable for drinking (9.09\%). The result of the study suggests that the groundwater of the area needs some degree of treatment before consumption, and it also needs to be protected from the threat of contamination. The groundwater is one of the major source of drinking water as well as for irrigation in study area.
\end{abstract}

Keywords: Arsenic, drinking water, physicochemical parameters, tube wells, turbidity

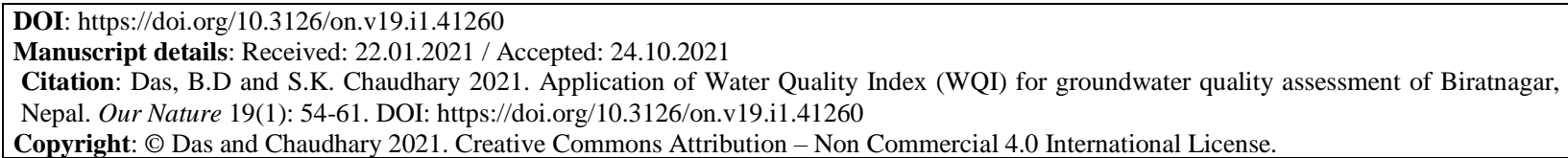

\section{Introduction}

Groundwater is the prime source of drinking water all over the world as they are often of good quality, difficult to pollute, widely distributed, and adjust throughout the year. Hydro-chemistry of groundwater may be influenced by multiple natural factors, such as chemical reactions between water and soil or sediments, biochemical reactions, and surface water - groundwater interactions, as well as human activities. The municipal as well as industrial wastewater, whether treated or not, is a permanent source of pollution that influences groundwater hydro-chemistry (UNEP/WHO, 1996). Additionally, groundwater may be affected by non-point sources of pollution, caused by surface and subsurface runoff from irrigation water and waste treatment plants in urban areas (Brunke and Gonser, 1997).

Water forms the world's streams, lakes, oceans and rain, and is the major constituent of the fluids of organisms which is an essential requirement of human and industrial developments 


\section{Das and Chaudhary / Our Nature | December 2021 | 19 (1): 54-61}

and it is one the most delicate part of the environment (Das and Acharya, 2003). Groundwater is used for domestic and industrial water supply and irrigation all over the world. Irrigated agricultural discharges into the groundwater can result about considerable change in the groundwater quality. These anthropogenic activities on the groundwater pose serious threat to the groundwater quality. If the groundwater is contaminated, its quality cannot be restored by stopping the pollutants from the sources. Therefore, it becomes crucial to regularly monitor the quality of groundwater and to device ways and means to protect it. A survey conducted in 1980, the WHO estimated that about 25 million people die every year from diseases caused by unsafe and inadequate drinking water and poor sanitary conditions (Agarwal, 1980).

Groundwater pollution has become a major subject of public concern the world over (Bockris, 1978). National Environmental Engineering Research Institute, Nagpur, India, reported that about $70 \%$ of the available water in India is polluted (Pani, 1986). Important water quality problems are found in the existing resources of Nepal and more than $70 \%$ of Nepal's population does not have access to clean, safe drinking water and an estimated $37 \%$ of the population does not

\section{Materials and methods}

\section{Study area}

Nepal, a small and landlocked country, ranged from the highest peak in the world to the plains of the Terai along the slopes of the Himalaya between China and India covering an area of $147,516 \mathrm{~km}^{2}$ being $800 \mathrm{~km}$ from east to west, and from $144 \mathrm{~km}$ to $240 \mathrm{~km}$ north to south, between $80^{\circ}-88^{\circ} \mathrm{E}$ and $26^{\circ}-31^{\circ} \mathrm{N}$ (Das and Choudhary, 2018). The country is bordered by India to the East, South and West and China to the North. The elevation ranges from $66 \mathrm{~m}$ to $8848 \mathrm{~m}$ above sea level (CBS, 2004). Nepal has seven physiographic divisions from south to north: Terai, Siwalik Hills, Mahabharat 'Lek' (Mountain range), Midlands, Himalayas, Inner Himalayas, and Tibetan marginal mountains (Hagen, 1998).

Biratnagar Metropolitan deceives in the plain area of Morang district of eastern Nepal (Fig. 1). even have access to the most rudimentary water treatment systems (World Resources Institute, 1999).

The Water Quality Index (WQI) is an individual numeric expression which clarifies the complex information obtained from any body of water, mostly related to water quality. WQI is an assessment reflecting the composite influence of different water quality parameters (Ramakrishnaiah et al., 2009), which is one of the most effective tools to communicate information on the quality of water to the concerned citizens and policy makers. It, therefore, becomes an important tool for the assessment and management of groundwater. Some studies on WQI such as from Nepal (Das et al., 2018; 2020; 2021), from India (Bhat and Pandit, 2014; Deep et al., 2020) have been carried out to assess the water quality.

Groundwater quality assessment ensures the sustainable safe use of water. On the basis of some very important water quality parameters, WQI can provide a simple indicator of water quality at a certain location and time. Therefore, this study has been carried out to assess the groundwater quality of Biratnagar in Morang District, Nepal, based on the analytical results of groundwater samples.

The city is situated in the south-west corner of Morang district at $26^{\circ} \square 23^{\prime}-26^{\circ} 30^{\prime} \mathrm{N}$ latitudes and $87^{\circ} 14^{\prime}-87^{\circ} 18^{\prime} \mathrm{E}$ longitudes. The city is bordered by Kesalia River in the west and north, Singhiya River in east and Jogbani (India) in south. Biratnagar is an industrial city of Nepal with many industries located in and around its suburbs which has traditionally been an agricultural hub and is home to many agriculture-based industries. The city comprises of 22 Administrative Wards and most of the people living in these wards depend on ground water sources i.e. shallow and deep tube wells for domestic purposes including drinking. The population of Biratnagar is 204,949 (Population Census, 2011). 


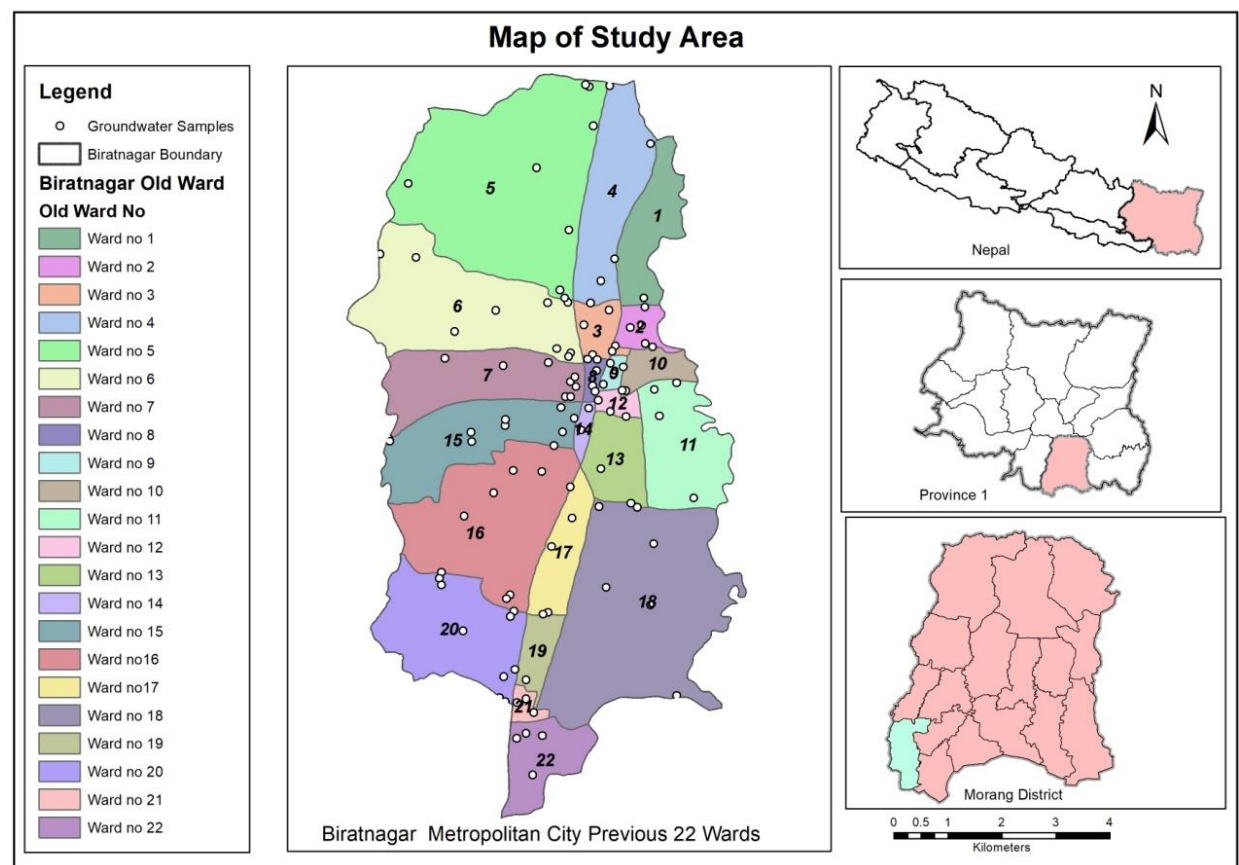

Figure 1. The map of Biratnagar Metropolitan showing water sampling sites in different wards.

\section{Sampling method}

Groundwater samples were collected from 110 representative tube wells ( 5 tube wells from each wards) during post-monsoon period of 2015. Sample locations were recorded using a Global Positioning System (GPS). Parameters like arsenic, fluoride, temperature, $\mathrm{pH}$, dissolved oxygen, freecarbon dioxide and conductivity were determined on the spot using field test kit (Conductiv TesterCD 98304) while the rest of the parameters were determined in the laboratory (University Department of Botany, Environmental Biology Research Laboratory, Tilka Manjhi Bhagalpur University, Bhagalpur, India). The overall analysis was done following the standard methods Trivedy and Goel (1986) and APHA (2012). The result was tabulated and compared to the given standard guidelines for drinking water (NDWQS, 2005; WHO, 2008).

\section{Calculation of WQI}

The WQI has been calculated to evaluate the suitability of groundwater quality of Biratnagar, Nepal for drinking purposes. The WHO standards for drinking purposes have been considered for the calculation of WQI (Trivedy and Goel, 1986). For the calculation of WQI, 10 parameters such as $\mathrm{pH}$, turbidity, electrical conductivity (EC), DO (dissolved oxygen), free-carbondioxide $\left(\mathrm{FCO}_{2}\right.$ ), bicarbonate $\left(\mathrm{HCO}_{3}\right)$, total hardness $(\mathrm{TH})$, phosphate $\left(\mathrm{PO}_{4}-\mathrm{P}\right)$, nitrate- $\mathrm{N}\left(\mathrm{NO}_{3}-\mathrm{N}\right)$ and arsenic (As) were analyzed.

All the analyzed parameters have been assigned weight (wi) according to their relative importance in the overall quality of water for drinking purposes (Table 1). The maximum weight of 5 has been assigned to parameters like nitrate and arsenic due to their major importance in water quality assessment (Ramakrishnaiah et al., 2009). Similarly, on the basis of their relative importance, the maximum weight of 4 has been assigned to $\mathrm{pH}$ and DO, the weight of 3 to free-carbondioxide $\left(\mathrm{FCO}_{2}\right)$ and bicarbonate $\left(\mathrm{HCO}_{3}\right)$ and weight of 2 to turbidity, electrical conductivity (EC), total hardness $(\mathrm{TH})$ and phosphate $\left(\mathrm{PO}_{4}-\mathrm{P}\right)$. The overall calculation of WQI has been carried out by the method given by Ramakrishnaiah, et al. (2009).

Table 1. Parameters applied to calculate the WQI and their Relative weight.

\begin{tabular}{cccc}
\hline Parameters/units & WHO standards & Weight (wi) & Relative weight (Wi) \\
\hline $\mathrm{pH}$ & $8.2-8.8$ & 4 & 0.125 \\
Turbidity NTU & 1.5 & 2 & 0.0625 \\
$\mathrm{EC} \mu \mathrm{S} / \mathrm{cm}$ & 1000 & 2 & 0.0625 \\
DO $\mathrm{mg} / \mathrm{L}$ & 5 & 4 & 0.125
\end{tabular}


Das and Chaudhary / Our Nature | December 2021 | 19 (1): 54-61

\begin{tabular}{cccc}
$\mathrm{FCO}_{2} \mathrm{mg} / \mathrm{L}$ & $6^{*}$ & 3 & 0.09375 \\
$\mathrm{HCO}_{3}{ }^{-} \mathrm{mg} / \mathrm{L}$ & $150-300^{*}$ & 3 & 0.09375 \\
$\mathrm{TH} \mathrm{mg} / \mathrm{L}$ & 500 & 2 & 0.0625 \\
$\mathrm{PO}_{4}{ }^{-} \mathrm{P} \mathrm{mg} / \mathrm{L}$ & $0.1-1.0$ & 2 & 0.0625 \\
$\mathrm{NO}_{3}{ }^{-\mathrm{N} \mathrm{mg} / \mathrm{L}}$ & 50 & 5 & 0.15625 \\
$\mathrm{Arsenic} \mathrm{mg/L}$ & $0.01-0.05$ & 5 & 0.15625 \\
\hline
\end{tabular}

*ISI Standard, all the standards are in $\mathrm{mg} / \mathrm{L}$ except $\mathrm{p}$, Turbidity and conductivity

Based on WQI value, the potable water of study area has been classified into 5 categories as excellent, good, poor, very poor and unfit for

\section{Results and discussion}

All the results for physicochemical parameters for post monsoon-2015 taken from a total of 110 sites were calculated with their mean value and standard deviation have been described below.

\section{Physicochemical analysis}

The temperature ranged from $19.84^{\circ} \mathrm{C} \pm 0.13$ (ward no. 22) to $22.34^{\circ} \mathrm{C} \pm 0.23$ (ward no. 7 ) and there was no WHO guideline value to be compared with. The $\mathrm{pH}$ value ranged from $7.5 \pm 0.21$ (ward no. 4) to $7.68 \pm 0.11$ (ward no. 9) and all the $\mathrm{pH}$ values were found within the range of WHO and NDWQS guidelines value. In the study, turbidity value ranged from $4.94 \pm 5.83$ (ward no. 14) to 81.46 \pm 44.28 NTU (ward no. 10). Regarding the turbidity values, during post monsoon $74(67.27 \%)$ out of 110 water samples were found to have crossed the WHO guideline limit of 5 NTU. The turbidity could be attributed to the presence of organic matter pollution, other effluents, run-off with high suspended particles and heavy rainfall (Chapman et al., 1996).

In the study, electrical conductivity (EC) value ranged from $277.8 \pm 55.12$ (ward no. 16) to $829.2 \pm 151.14 \mu \mathrm{S} / \mathrm{cm}$ (ward no. 12) and all the values were under the WHO guideline limit of 1000 drinking purposes following Ramakrishnaiah et al. (2009).

$\mu \mathrm{S} / \mathrm{cm}$ stipulated for drinking and domestic water. The $\mathrm{FCO}_{2}$ value ranged from 13.20 \pm 1.10 (ward no. 1) to $17.6 \pm 3.58 \mathrm{mg} / \mathrm{L}$ (ward no. 10) and all the values were under the WHO guideline. The bicarbonate $\left(\mathrm{HCO}_{3}\right)$ value ranged from $24 \pm 3.74$ (ward no. 1) to $152.8 \pm 33.90 \mathrm{mg} / \mathrm{L}$ (ward no. 10), all the values were under the WHO guideline. The value of total hardness ranged from $79.60 \pm 12.68$ (ward no. 1) to $1942 \pm 3990.54 \mathrm{mg} / \mathrm{L}$ (ward no. 21) and all the values were under the WHO guideline. The phosphate values ranged from $0.15 \pm 0.00$ (ward no. 13 ) to $0.17 \pm 0.01 \mathrm{mg} / \mathrm{L}$ (ward no. 3) whereas value of nitrate- $\mathrm{N}\left(\mathrm{NO}_{3}-\mathrm{N}\right)$, ranged from $0.34 \pm 0.00$ (ward no. 1) to $0.39 \pm 0.04 \mathrm{mg} / \mathrm{L}$ (ward no. 3 ) and all the values of both were under the WHO guideline.

The value of arsenic (As) ranged from $0.01 \pm 0.00$ (ward no. 3) to $0.28 \pm 0.32 \mathrm{mg} / \mathrm{L}$ (ward no. 12). The very low concentration of As (below detection limit) was also noticed throughout the study. However, in 42 (38.18\%) out of 110 water samples, arsenic concentrations exceeded the WHO guideline limit of $0.01 \mathrm{mg} / \mathrm{L}$. The status of fluoride was below detectable level (BDL) in all the analyzed groundwater samples.

In this study, the computed WQI values ranges from 84.54 to 403.14 (Table 2, Fig. 2).

\section{WQI of Groundwater Samples of Biratnagar, Nepal}

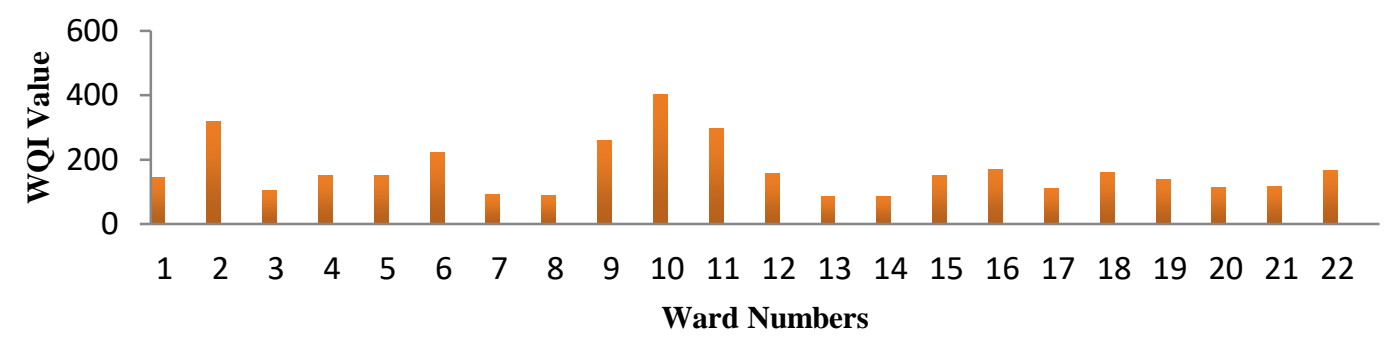

Figure 2. Water Quality Index (WQI) of groundwater samples of Biratnagar city, Nepal. 


\section{Das and Chaudhary / Our Nature | December 2021 || 19 (1): 54-61}

Table 2. Quality rating (Qi), Sub-index of each chemical parameter (SIi), WQI and water classification of each groundwater sample of Biratnagar, Nepal.

\begin{tabular}{|c|c|c|c|c|c|c|c|c|c|c|c|c|c|c|c|c|c|c|c|c|c|c|}
\hline \multirow{2}{*}{$\begin{array}{l}\text { Ward } \\
\text { No. }\end{array}$} & \multicolumn{2}{|l|}{ pH } & \multicolumn{2}{|c|}{ Turb. NTU } & \multicolumn{2}{|c|}{$\begin{array}{l}\text { E.C. } \mu \mathrm{s} / \mathrm{c} \\
\mathrm{m}\end{array}$} & \multicolumn{2}{|c|}{$\begin{array}{l}\text { DO } \\
\mathrm{mg} / \mathrm{L}\end{array}$} & \multicolumn{2}{|c|}{$\mathrm{FCO}_{2} \mathrm{mg} / \mathrm{L}$} & \multicolumn{2}{|c|}{$\begin{array}{l}\mathrm{HCO}_{3}^{-} \\
\mathrm{mg} / \mathrm{L} \\
\end{array}$} & \multicolumn{2}{|c|}{ T.H. mg/L } & \multicolumn{2}{|c|}{$\begin{array}{l}\mathrm{PO}_{4}{ }^{-P} \\
\mathrm{mg} / \mathrm{L}\end{array}$} & \multicolumn{2}{|c|}{$\begin{array}{l}\mathrm{NO}_{3}{ }^{-} \mathrm{N} \\
\mathrm{mg} / \mathrm{L}\end{array}$} & \multicolumn{2}{|c|}{ As $\mathrm{mg} / \mathrm{L}$} & \multirow{2}{*}{$\begin{array}{l}\text { WQI } \\
\text { Value }\end{array}$} & \multirow{2}{*}{$\begin{array}{l}\text { Class } \\
\text { of } \\
\text { Water }\end{array}$} \\
\hline & $\mathbf{Q i}$ & SIi & i & Ii & Qi & SIi & Qi & SIi & $\mathbf{Q i}$ & SIi & Qi & SIi & Qi & SIi & $\mathbf{Q i}$ & SIi & Qi & SIi & Qi & SIi & & \\
\hline 1 & $\begin{array}{l}89.1 \\
8\end{array}$ & $\begin{array}{l}11.1 \\
5\end{array}$ & 27.44 & 1.65 & $\begin{array}{l}63.2 \\
6\end{array}$ & $\begin{array}{l}3.9 \\
2\end{array}$ & $\begin{array}{l}33 . \\
6\end{array}$ & $\begin{array}{l}4 . \\
2\end{array}$ & 220.0 & $\begin{array}{l}20.4 \\
6\end{array}$ & 25.96 & 2.41 & 15.92 & 0.06 & 170.0 & 10.62 & 0.68 & 0.11 & $\begin{array}{l}566.6 \\
7\end{array}$ & $\begin{array}{l}88.5 \\
4\end{array}$ & $\begin{array}{l}143.1 \\
2\end{array}$ & Poor \\
\hline 2 & \begin{tabular}{|l}
89.8 \\
8 \\
\end{tabular} & \begin{tabular}{|l}
11.2 \\
4 \\
\end{tabular} & 3340.0 & 200.4 & $\begin{array}{l}65.6 \\
2 \\
\end{array}$ & $\begin{array}{l}3.9 \\
4 \\
\end{array}$ & $\begin{array}{l}32 . \\
8 \\
\end{array}$ & $\begin{array}{l}4 . \\
1 \\
\end{array}$ & 240.0 & $\begin{array}{l}22.3 \\
2 \\
\end{array}$ & 24.53 & 2.28 & 20.08 & 1.20 & 170 & 10.62 & 0.72 & 0.11 & 400.0 & 62.5 & $\begin{array}{l}318.7 \\
1 \\
\end{array}$ & Unfit \\
\hline 3 & \begin{tabular}{|l|}
89.8 \\
8 \\
\end{tabular} & \begin{tabular}{|l|}
11.2 \\
4 \\
\end{tabular} & 740.0 & 46.25 & $\begin{array}{l}72.6 \\
2 \\
\end{array}$ & $\begin{array}{l}4.5 \\
4 \\
\end{array}$ & $\begin{array}{l}37 . \\
6 \\
\end{array}$ & $\begin{array}{l}4 . \\
7\end{array}$ & 46.6 & $\begin{array}{l}23.1 \\
1 \\
\end{array}$ & 27.38 & 2.57 & 24.0 & 1.5 & 170 & 10.62 & 0.78 & .12 & 00 & 00 & \begin{tabular}{|l|}
104.6 \\
5
\end{tabular} & Poor \\
\hline 4 & $\begin{array}{l}88.2 \\
4 \\
\end{array}$ & $\begin{array}{l}11.0 \\
3 \\
\end{array}$ & 47 & 167 & 59.7 & $\begin{array}{l}3.7 \\
3\end{array}$ & $\begin{array}{l}34 . \\
4\end{array}$ & $\begin{array}{l}4 . \\
3\end{array}$ & 50.0 & $\begin{array}{l}23.4 \\
4 \\
\end{array}$ & 26.84 & 2.52 & 5.68 & 1.61 & 170 & 10.62 & 0.7 & 0.11 & 6.67 & 1.41 & $\begin{array}{l}150.4 \\
4\end{array}$ & Poor \\
\hline 5 & $\begin{array}{l}88.9 \\
4 \\
\end{array}$ & $\begin{array}{l}11.1 \\
2\end{array}$ & 73.3 & 2.08 & $\begin{array}{l}44.1 \\
8\end{array}$ & $\begin{array}{l}2.7 \\
6\end{array}$ & 36. & $\begin{array}{l}4 . \\
6\end{array}$ & 46.6 & $\begin{array}{l}23.1 \\
1\end{array}$ & 22.58 & 2.17 & 1.04 & 1.32 & 170 & 0.62 & 0.7 & .11 & 6.67 & .41 & 49.3 & Poor \\
\hline 6 & $\begin{array}{l}88.9 \\
4 \\
\end{array}$ & $\begin{array}{l}11.1 \\
2 \\
\end{array}$ & $\begin{array}{l}2317.3 \\
3\end{array}$ & 144.8 & $\begin{array}{l}39.9 \\
6 \\
\end{array}$ & 2.5 & $\begin{array}{l}33 . \\
6\end{array}$ & $\begin{array}{l}4 . \\
2\end{array}$ & 240.0 & $\begin{array}{l}22.3 \\
2 \\
\end{array}$ & 51.56 & 4.83 & 23.2 & 1.45 & 170 & 10.62 & 0.7 & 0.11 & 33.3 & \begin{tabular}{|l|}
20.8 \\
3 \\
\end{tabular} & $\begin{array}{l}222.8 \\
1 \\
\end{array}$ & V. Poo \\
\hline 7 & $\begin{array}{l}88.9 \\
4 \\
\end{array}$ & \begin{tabular}{|l|}
11.1 \\
2 \\
\end{tabular} & 512.0 & 32.0 & $\begin{array}{l}46.8 \\
2 \\
\end{array}$ & $\begin{array}{l}2.9 \\
3 \\
\end{array}$ & $\begin{array}{l}36 . \\
8 \\
\end{array}$ & $\begin{array}{l}4 . \\
6\end{array}$ & 40.0 & $\begin{array}{l}22.3 \\
2 \\
\end{array}$ & 53.69 & 5.03 & 24.16 & 1.51 & 170 & 10.62 & 0.7 & 0.11 & 66.67 & 1.41 & 91.65 & Good \\
\hline 8 & \begin{tabular}{|l|}
89.1 \\
8 \\
\end{tabular} & \begin{tabular}{|l|}
11.1 \\
5 \\
\end{tabular} & 100 & .75 & 67.1 & $\begin{array}{l}4.2 \\
0 \\
\end{array}$ & $\begin{array}{l}35 . \\
2\end{array}$ & $\begin{array}{l}4 . \\
4\end{array}$ & $\begin{array}{l}266.6 \\
7 \\
\end{array}$ & 250 & 61.33 & .75 & 27.6 & 1.73 & 170 & 10.62 & 0.72 & .11 & 100.0 & \begin{tabular}{|l|}
15.6 \\
3 \\
\end{tabular} & 37.34 & Good \\
\hline 9 & $\begin{array}{l}90.3 \\
5 \\
\end{array}$ & $\begin{array}{l}11.2 \\
9 \\
\end{array}$ & 29.3 & $\begin{array}{l}120.5 \\
8 \\
\end{array}$ & 75.6 & $\begin{array}{l}4.7 \\
3 \\
\end{array}$ & $\begin{array}{l}32 . \\
8\end{array}$ & $\begin{array}{l}4 . \\
1\end{array}$ & $\begin{array}{l}286.6 \\
7 \\
\end{array}$ & $\begin{array}{l}26.8 \\
8 \\
\end{array}$ & 67.56 & .33 & 0.4 & .9 & 170 & 10.62 & 0.7 & 0.11 & 66.6 & \begin{tabular}{|l|}
72.9 \\
2 \\
\end{tabular} & \begin{tabular}{|l|}
259.4 \\
6 \\
\end{tabular} & V. Poor \\
\hline 10 & $\begin{array}{l}89.4 \\
1\end{array}$ & $\begin{array}{l}11.7 \\
8\end{array}$ & $\begin{array}{l}5430.6 \\
7\end{array}$ & 339.4 & 57.9 & $\begin{array}{l}3.6 \\
2\end{array}$ & 36. & $\begin{array}{l}4 . \\
6\end{array}$ & $\begin{array}{l}293.3 \\
3\end{array}$ & 27.5 & 28.27 & 2.65 & 2.8 & 1.43 & 170 & 10.62 & 0.72 & 0.11 & 66.67 & 1.41 & $\begin{array}{l}403.1 \\
4\end{array}$ & Unfit \\
\hline 11 & $\begin{array}{l}88.9 \\
4 \\
\end{array}$ & $\begin{array}{l}11.1 \\
2\end{array}$ & 3816.0 & 238.5 & $\begin{array}{l}41.5 \\
4 \\
\end{array}$ & 2.6 & $\begin{array}{l}38 . \\
4\end{array}$ & $\begin{array}{l}4 . \\
8\end{array}$ & 246.6 & $\begin{array}{l}23.1 \\
1 \\
\end{array}$ & 17.6 & 1.65 & 35.36 & 2.21 & 170 & 10.62 & 0.72 & 0.11 & 66.67 & 1.41 & $\begin{array}{l}296.1 \\
3 \\
\end{array}$ & V. Poor \\
\hline 12 & $\begin{array}{l}88.9 \\
4 \\
\end{array}$ & \begin{tabular}{|l|}
11.1 \\
2 \\
\end{tabular} & 573.3 & 8.33 & $\begin{array}{l}82.9 \\
2 \\
\end{array}$ & 5.2 & $\begin{array}{l}35 . \\
2 \\
\end{array}$ & $\begin{array}{l}4 . \\
4\end{array}$ & 260.0 & $\begin{array}{l}24.3 \\
8 \\
\end{array}$ & 28.27 & 2.65 & 27.84 & 1.74 & 150 & 9.38 & 0.72 & 0.11 & 00 & 00 & 157.3 & Poor \\
\hline 13 & $\begin{array}{l}88.4 \\
7 \\
\end{array}$ & $\begin{array}{l}11.0 \\
6 \\
\end{array}$ & 374.67 & 23.42 & $\begin{array}{l}66.1 \\
6 \\
\end{array}$ & 4.1 & $\begin{array}{l}36 . \\
0 \\
\end{array}$ & $\begin{array}{l}4 . \\
5\end{array}$ & $\begin{array}{l}253.3 \\
3 \\
\end{array}$ & $\begin{array}{l}23.7 \\
5 \\
\end{array}$ & 24.0 & 2.25 & 36.0 & 2.25 & 150 & 9.38 & 0.7 & 0.11 & 33.33 & 5.21 & 86.07 & Good \\
\hline 14 & $\begin{array}{l}88.7 \\
1 \\
\end{array}$ & $\begin{array}{l}11.0 \\
9 \\
\end{array}$ & 329.33 & 20.58 & $\begin{array}{l}81.0 \\
8 \\
\end{array}$ & 5.1 & $\begin{array}{l}33 . \\
6\end{array}$ & $\begin{array}{l}4 . \\
2\end{array}$ & 260.0 & $\begin{array}{l}24.3 \\
8 \\
\end{array}$ & 27.56 & 2.58 & 30.56 & 1.91 & 150 & 9.38 & 0.7 & 0.11 & 33.33 & 5.21 & 84.54 & Good \\
\hline 15 & 88.7 & 11.0 & 1394.6 & 7.17 & 56.7 & 3.5 & 34. & 4. & 266.6 & 25.0 & 19.91 & 1.87 & 30.56 & 1.91 & 150 & 9.38 & 0.72 & 0.11 & 33.33 & 5.21 & 149.5 & Poor \\
\hline
\end{tabular}


Das and Chaudhary / Our Nature | December 2021 | 19 (1): 54-61

\begin{tabular}{|c|c|c|c|c|c|c|c|c|c|c|c|c|c|c|c|c|c|c|c|c|c|c|}
\hline & 1 & 9 & 7 & & 6 & 5 & 4 & 3 & 7 & & & & & & & & & & & & 9 & \\
\hline 16 & $\begin{array}{l}89.1 \\
8 \\
\end{array}$ & $\begin{array}{l}11.1 \\
5 \\
\end{array}$ & $\begin{array}{l}1729.3 \\
3\end{array}$ & \begin{tabular}{|l|}
108.0 \\
8 \\
\end{tabular} & $\begin{array}{l}27.7 \\
8 \\
\end{array}$ & $\begin{array}{l}1.7 \\
4 \\
\end{array}$ & $\begin{array}{l}34 . \\
4 \\
\end{array}$ & $\begin{array}{l}4 . \\
3\end{array}$ & 280.0 & $\begin{array}{l}26.2 \\
5 \\
\end{array}$ & 10.67 & 1.0 & 25.12 & 1.57 & 150 & 9.38 & 0.68 & O.11 & 33.33 & 5.21 & $\begin{array}{l}168.6 \\
8 \\
\end{array}$ & Poor \\
\hline 17 & \begin{tabular}{|l|}
89.4 \\
1 \\
\end{tabular} & $\begin{array}{l}11.7 \\
8 \\
\end{array}$ & 844.0 & 52.75 & $\begin{array}{l}34.8 \\
8 \\
\end{array}$ & $\begin{array}{l}2.1 \\
8 \\
\end{array}$ & \begin{tabular}{|l}
35. \\
2 \\
\end{tabular} & $\begin{array}{l}4 . \\
4\end{array}$ & $\begin{array}{l}286.6 \\
7 \\
\end{array}$ & $\begin{array}{l}26.8 \\
8 \\
\end{array}$ & 11.73 & 1.1 & 20.56 & 1.29 & 160 & 10.0 & 0.6 & 0.1 & 00 & 00 & \begin{tabular}{|l|}
110.4 \\
8 \\
\end{tabular} & Poor \\
\hline 18 & $\begin{array}{l}88.9 \\
4\end{array}$ & $\begin{array}{l}11.1 \\
2 \\
\end{array}$ & $\begin{array}{l}1630.6 \\
7\end{array}$ & $\begin{array}{l}101.9 \\
2 \\
\end{array}$ & $\begin{array}{l}36.3 \\
6 \\
\end{array}$ & $\begin{array}{l}2.2 \\
7\end{array}$ & $\begin{array}{l}36 . \\
8\end{array}$ & $\begin{array}{l}4 . \\
6\end{array}$ & 240.0 & $\begin{array}{l}22.3 \\
2 \\
\end{array}$ & 12.8 & 1.2 & 17.2 & 1.1 & 17 & 10.62 & 0.7 & 0.11 & 33.33 & 5.21 & $\begin{array}{l}160.4 \\
7\end{array}$ & Poor \\
\hline 19 & \begin{tabular}{|l}
89.4 \\
1 \\
\end{tabular} & $\begin{array}{l}11.7 \\
8\end{array}$ & $\begin{array}{l}1298.6 \\
7\end{array}$ & 81.17 & $\begin{array}{l}42.4 \\
6 \\
\end{array}$ & $\begin{array}{l}2.6 \\
5 \\
\end{array}$ & $\begin{array}{l}36 . \\
8 \\
\end{array}$ & $\begin{array}{l}4 . \\
6\end{array}$ & 240.0 & $\begin{array}{l}22.3 \\
2 \\
\end{array}$ & 13.51 & 1.27 & 24.4 & 1.53 & 160 & 10.0 & 0.7 & 0.11 & 66.67 & 1.41 & \begin{tabular}{|l|}
136.8 \\
4 \\
\end{tabular} & Poor \\
\hline 20 & \begin{tabular}{|l|}
88.9 \\
4 \\
\end{tabular} & $\begin{array}{l}11.1 \\
2 \\
\end{array}$ & 897.33 & 56.08 & $\begin{array}{l}32.1 \\
2 \\
\end{array}$ & 2.0 & $\begin{array}{l}36 . \\
0 \\
\end{array}$ & $\begin{array}{l}4 . \\
5\end{array}$ & $\begin{array}{l}246.6 \\
7 \\
\end{array}$ & $\begin{array}{l}23.1 \\
1 \\
\end{array}$ & 12.8 & 1.2 & 18.4 & 1.15 & 160 & 10.0 & 0.7 & 0.11 & 33.33 & 5.21 & \begin{tabular}{|l|}
114.4 \\
8 \\
\end{tabular} & Poor \\
\hline 21 & \begin{tabular}{|l|}
89.6 \\
5 \\
\end{tabular} & $\begin{array}{l}11.2 \\
1 \\
\end{array}$ & 917.33 & 57.33 & $\begin{array}{l}57.4 \\
2 \\
\end{array}$ & $\begin{array}{l}3.5 \\
9 \\
\end{array}$ & $\begin{array}{l}33 . \\
6 \\
\end{array}$ & $\begin{array}{l}4 . \\
2\end{array}$ & $\begin{array}{l}233.3 \\
3 \\
\end{array}$ & $\begin{array}{l}21.8 \\
8 \\
\end{array}$ & 17.1 & 1.6 & 28.8 & 1.8 & 170 & 10.62 & 0.7 & 0.11 & 33.33 & 5.21 & $\begin{array}{l}117.5 \\
5 \\
\end{array}$ & Poor \\
\hline 22 & \begin{tabular}{|l|}
88.9 \\
4 \\
\end{tabular} & $\begin{array}{l}11.1 \\
2 \\
\end{array}$ & 1820.0 & \begin{tabular}{|l|}
113.7 \\
5 \\
\end{tabular} & $\begin{array}{l}54.6 \\
2 \\
\end{array}$ & $\begin{array}{l}3.3 \\
1 \\
\end{array}$ & $\begin{array}{l}34 . \\
4 \\
\end{array}$ & $\begin{array}{l}4 . \\
3\end{array}$ & 220.0 & $\begin{array}{l}20.6 \\
3 \\
\end{array}$ & 14.04 & 1.32 & 21.2 & 1.33 & 160 & 10.0 & 0.7 & 0.11 & 66.67 & 1.41 & $\begin{array}{l}167.2 \\
8 \\
\end{array}$ & Poor \\
\hline
\end{tabular}


Das and Chaudhary / Our Nature | December 2021 | 19 (1): 54-61

Table 3. Water quality classification of different ward nos. of Biratnagar city based on WQI value

\begin{tabular}{|c|c|c|c|c|}
\hline WQI value & $\begin{array}{l}\text { Water } \\
\text { Category }\end{array}$ & \% of water samples & Ward no & Class \\
\hline $\begin{array}{l}< \\
50\end{array}$ & Excellent water & 0 & 0 & $\mathrm{~A}$ \\
\hline $50.1-100$ & Good water & 18.18 & $7,8,13,14$ & $\mathrm{~B}$ \\
\hline $100.1-200$ & Poor water & 59.09 & $1,3,4,5,12,15-22$ & $\mathrm{C}$ \\
\hline $200-300$ & Very poor water & 13.64 & $6,9,11$ & $\mathrm{D}$ \\
\hline$>300$ & Unsuitable for drinking & 9.09 & 2,10 & $\mathrm{E}$ \\
\hline
\end{tabular}

Based on WQI values (Tables 2, 3), no any ward has excellent quality of water whereas $18.18 \%$ of good quality (from ward no. 7, 8, 13 and 14), $59.09 \%$ of poor quality (from ward no. $1,3,4,5,12$ and $15-22$ ), $13.64 \%$ of very poor quality (from ward no. 6, 9 and 11) and $9.09 \%$ of unsuitable for drinking (from ward no. 2 and 10) have been reported. It showed that ground water of ward no. $1,2,3,4,5,6,9,10,11,12$ and $15-22$ of Biratnagar are not suitable for drinking and the water from ward number 7, 8, 13 and 14 are safe and suitable for drinking according to WHO limits.

\section{Conclusion}

In the present study, the WQI for the groundwater samples of Biratnagar ranges from 84.54 to 403.14. The value of WQI has been influenced mainly due to the higher sub-index (SIi) values of turbidity, $\mathrm{FCO}_{2}$ and arsenic in the groundwater. The results of investigation have been used to suggest models for predicting water quality. Application of WQI in this study has been found very useful in the assessment of the overall groundwater quality and management of Biratnagar city.

According to findings, no any ward had excellent (class A) quality of water. Among all, good quality of water (class B) was reported only from ward no.

\section{References}

Agarwal, A. 1980. A decade of clean water. News Scientist 88-1226.

APHA 2012. Standard Methods for Examination of Water and Waste water. 22th Edn. American Public Health Association, Washington DC, USA, 1220.

Bhat, S.A. and A.K. Pandit 2014. Surface water quality assessment of Wular Lake, A Ramsar site in Kashmir Himalaya, using Discriminant Analysis and WQI. Journal of Ecosystems $\quad 2014(1): \quad 1-18$. http://dx.doi.org/10.1155/2014/724728

Bockris, J. O’M. 1978. Environmental chemistry. Premium Press, New York. 332p.
7,8 and 13 whereas poor quality of water (class C) was reported from ward no. 1, 3, 4, 5, 12 and 15 to 22; very poor quality (class D) from ward no. 6, 9 and 11; and unsuitable for drinking water (class E) from ward no. 2 and 10. The highly contaminated ground water was found in ward no. 2 and 19 and least contaminated was found in ward no. 7, 8, 13 and 14. The reasons of contamination for arsenic is geogenic whereas for others was basically anthropogenic, i.e., high populated area, lack of proper drainage and waste disposal system.

Groundwater is the main source for drinking, domestic and irrigation uses in the study area. The result of the study strongly suggests and recommend to the authority that the groundwater of the area needs some degree of treatment for drinking purpose and it also needs to be protected from the threat of contamination.

\section{Acknowledgments}

The authors are grateful to the University Department of Botany, T.M. Bhagalpur University, Bhagalpur, Bihar, India; Department of Botany, Mahendra Morang Aadarsh Multiple Campus, Tribhuvan University, Biratnagar, Nepal; and Nepal Environmental Service Centre, Biratnagar for providing laboratory facilities.

Brunke, M., and T. Gonser 2003. The ecological significance of exchange processes between rivers and groundwater. Freshwater Biology 37(1): 1-33. https://doi.org/10.1046/j.13652427.1997.00143.x

CBS 2004. Environment statistics of Nepal. Kathmandu, Nepal. Central Bureau of Statistics (CBS), Kathmandu, Nepal.

Chapman, D.V., World Health Oganization, UNESCO, and United Nations Environment Programme 1996. Water quality assessment: A guide to the use of biota, sediments and water in environmental monitoring / edited by 
Deborah Chapman (2 ${ }^{\text {nd }}$ ed.). E and FN Spon, London.

Das, B. D., and S. K. Choudhary 2018. Seasonal variations in physicochemical properties with trace metals and microbial status in groundwaters of Biratnagar, Nepal, Journal of Emerging Technologies and Innovative Research 5(7): 153-166.

Das, B. D., B. N. Kumar, R. K. Mishra, and S. K. Choudhary 2018. Assessment of Water Quality Index for the surface water in Betna Wetland of Morang district, Nepal. Journal of Emerging Technologies and Innovative Research 5(2): 831-834.

Das, B. D., R. K. Mishra, A. Kumar, R. Kumari, V. Das, N. Paudel and S. K. Choudhary 2020. Assessment of surface water quality by using Water Quality Index of Sanbarish Pond of Morang district, Nepal. American Scientific Research Journal for Engineering, Technology, and Sciences 63(1): 137-143.

Das, B. D., R. K. Mishra, and S. K. Choudhary 2021. Groundwater quality in Biratnagar of Morang district, Nepal. International Journal of Research - GRANTHAALAYAH 9(5): 368-377. https://doi.org/10.29121/granthaalayah.v9.i 5.2021.3961

Das, J. and B. C. Acharya 2003. Hydrology and assessment of lotic water quality in Cuttack city, India. Water, Air, Soil Pollution 150: 163-175. https://doi.org/10.1023/A: 1026193514875

Deep, A., V. Gupta, L. Bisht, and R. Kumar 2020. Application of WQI for water quality assessment of high-altitude snow-fed sacred Lake Hemkund, Garhwal Himalaya. Sustainable Water Resources Management 6(5): 89. https://doi.org/10.1007/s40899-020-00449$\mathrm{W}$

Hagen, T. 1998. Nepal - the Kingdom in the Himalaya $\left(4^{\text {th }}\right.$ ed.). Himal Books, Lalitpur, Nepal.
NDWQS 2005. National drinking water quality standards, 2005, Implementation directives for National Drinking Water Quality Standards, 2062. Ministry of Physical Planning and Works, Government of Nepal, Singhadarbar, Kathmandu, Nepal.

Pani, B. S. 1986. Outfall diffusers. In Abstract of the National Seminar on Air and Water Pollution. April, University College of Engineering, Burla.

Population Census 2011. National report to the Government of Nepal by the National Planning Commission Secretariat. Central Bureau of Statistics (CBS), Kathmandu, Nepal.

Ramakrishnaiah, C. R., C. Sadashivaiah, and G. Ranganna 2009. Assessment of Water Quality Index for the groundwater in Tumkur Taluk, Karnataka State. Indian Journal of Chemistry 6: 523-530. https://doi.org/10.1155/2009/757424

Trivedy, R. K. and P. K. Goel 1986. Chemical and biological methods for water pollution studies. Environmental Publication (Karad, India) 6: 10-12.

UNEP/WHO 1996. Water quality monitoring - A practical guide to the design and implementation of freshwater quality studies and monitoring programmes. Published on behalf of United Nations Environment Program and the World Health Organization (C) 1996 UNEP/WHO ISBN 0419223207 (Hbk) 0419217304 (Pbk)

WHO 2008. Guidelines for drinking-water quality $\left(3^{\text {rd }}\right.$ ed.). WHO Press, World Health Organization, 20 Avenue Appia, 1211 Geneva 27, Switzerland.

World Resources Institute 1999. http://www.wri.org/facts/data-tables.html 